\title{
Editorial: Brain Insulin Resistance in Neurodevelopmental and Neurodegenerative Disorders: Mind the Gap!
}

\author{
Mara Dierssen 1,2,3,4 and Eugenio Barone ${ }^{5 *}$ \\ ${ }^{1}$ Centre for Genomic Regulation (CRG), The Barcelona Institute of Science and Technology, Barcelona, Spain, ${ }^{2}$ Department \\ of Experimental Sciences, Universitat Pompeu Fabra (UPF), Barcelona, Spain, ${ }^{3}$ Human Pharmacology and Clinical \\ Neurosciences Research Group, Neurosciences Research Program, Hospital Del Mar Medical Research Institute (IMIM), \\ Barcelona, Spain, ${ }^{4}$ Centro de Investigación Biomédica en Red de Enfermedades Raras (CIBERER), Valencia, Spain, \\ ${ }^{5}$ Department of Biochemical Sciences "A. Rossi-Fanelli", Sapienza University of Rome, Rome, Italy
}

Keywords: insulin resistance, neurodevelopment and brain metabolism, neurodegenerative diseases, learning and memory, brain insulin resistance, Alzheimer's disease, Down syndrome, Parkinson's disease

\section{Editorial on the Research Topic}

Brain Insulin Resistance in Neurodevelopmental and Neurodegenerative Disorders: Mind the Gap!

The failure of insulin signaling-a condition known as insulin resistance-is a key pathological feature of both type 2 diabetes (T2DM, systemic insulin resistance) (Arnold et al., 2018; Kellar and Craft, 2020) and brain disorders, such as Alzheimer disease and related dementias (ADRDs, brain insulin resistance) but also other neurodegenerative disorders such as Parkinson's or Huntington's disease (Brás et al., 2019; Hong et al., 2020), neurodevelopmental disorders such as Down syndrome (DS) (Tramutola et al., 2020; Lanzillotta et al., 2021) or autism (Manco et al., 2021) and behavioral disorders (Kleinridders et al., 2015), and greatly contributes to their pathogenesis (Arnold et al., 2018; Kellar and Craft, 2020). Specifically, regarding ADRDs and T2DM, considerable overlap has been found in the risk factors, comorbidities and putative pathophysiological mechanisms, leading to the proposal that AD is type 3 diabetes (Butterfield et al., 2014; de la Monte, 2019). Examination of postmortem AD, amnestic mild cognitive impairment and DS brains, uncovered key signs of brain insulin resistance, i.e., reduced insulin receptor (IR) and increased serine phosphorylation (inhibitory) of insulin receptor substrate 1 (IRS1), as well as reduced activation of pathways downstream from IRS1, particularly in the hippocampus, cortex, and hypothalamus (Talbot et al., 2012; Tramutola et al., 2015, 2020; Sharma et al., 2019). Higher levels of insulin resistance markers are associated with poorer performance on cognitive tests of episodic and working memory, independent of the load of senile plaques and tangles, thus suggesting a role for insulin signaling in neuronal functions (Talbot et al., 2012). At the cellular level, these dysfunctions might manifest as the impairment of neuroplasticity, receptor regulation or neurotransmitter release in neurons (Spinelli et al., 2017; Barone et al., 2019; Franklin et al., 2019; Melo et al., 2020; Lanzillotta et al., 2021), or the impairment of processes more directly implicated in insulin metabolism, such as neuronal glucose uptake in neurons expressing GLUT4, or homeostatic or inflammatory responses to insulin (Bomfim et al., 2012; Lourenco et al., 2013; Barone et al., 2016; Triani et al., 2018; Melo et al., 2020; Lanzillotta et al., 2021). Further, intense research over the last two decades has highlighted the impact of insulin signaling, brain energy balance, and their 
fluctuations on neurogenesis processes both during postnatal and adult life (Barone et al., 2014; Arnold et al., 2018; Kellar and Craft, 2020). Indeed, diabetes, obesity, and overweight are prevalent pregnancy complications that predispose offspring to neurodevelopmental disorders (Fusco et al., 2019; Dearden et al., 2020). Moreover, impaired neurogenesis compromises hippocampal function and plays a role in cognitive deficits in ADDRs (Arnold et al., 2018). In those plastic neural tissues, activation of insulin signaling regulates birth, specification, migration, and integration of newly generated neurons, suggesting that alterations of this key signaling transduction pathway may have a role both in neurodevelopmental disorders and adult neurogenesis (Arnold et al., 2018; Kellar and Craft, 2020).

The aim of this Research Topic is to outline the major needs and challenges in the comprehension of the role of insulin signaling and its alterations in the brain and how such alterations contribute to the development of neurodevelopmental and neurodegenerative disorders.

Recently, several studies evaluated the effects of nutrientrelated signals on both brain development and cognitive functions. A key finding was the discovery that, other than hypothalamus, a number of brain regions express receptors for hormones known to regulate metabolic processes. In particular, insulin signaling was found to impact on molecular pathways underlying hippocampal plasticity, learning, and memory. Spinelli et al. discuss evidence linking the altered insulin sensitivity in the hippocampus with defects of both adult neurogenesis and synaptic plasticity. These authors also review epidemiological studies and the observations collected in experimental models focusing the attention on the critical role of brain insulin resistance at the crossroad between metabolic and neurodegenerative diseases.

One of the key questions regarding the effects of insulin within the brain, has to do with insulin uptake. Indeed, two sources for insulin in the brain have been identified: (1) circulating insulin produced by the pancreas enters into the brain through a receptor-mediated uptake at the level of blood brain barrier (BBB) (Rhea and Banks; Rhea et al., 2020); or (2) in situ synthesis by specific neuronal populations (Nakabeppu, 2019). While the second hypothesis is still under debate, the first one is the most accepted and validated by several studies. Rhea and Banks provide a comprehensive review about the role of the different cell types found at the BBB in regulating insulin uptake. Furthermore, they discuss how alterations of BBB favor the development of brain insulin resistance by stressing the role for the IR at the BBB, that goes beyond its canonical role in mediating the activation of the signal. Indeed, IR at the BBB is the main transporter for insulin within the brain. In this context, the review also addresses the effects of intranasal insulin administration and weight loss-associated improved insulin sensitivity, that are two validated strategies to increase brain insulin uptake and promoting neuroprotective effects.

From a molecular point of view, the activation of the insulin signaling requires IR-mediated tyrosine (Tyr) phosphorylation of IRS1. Following IRS1 activation, two main signaling cascades are activated downstream from IRS1: the mitogen-activated protein kinase (MAPK), and the phosphatidyl-inositol 3-kinase $(\mathrm{PI} 3 \mathrm{~K}) /$ protein kinase B (Akt). In the brain, MAPK are involved in the regulation of genes controlling synapse growth, neuronal maintenance, and repair processes while the PI3K/Akt pathway is involved in the maintenance of synaptic plasticity, stress response and neuronal metabolism and autophagy (Tramutola et al., 2016; Arnold et al., 2018). Gabbouji et al. highlight the role for the PI3K/Akt axis in the brain, showing how similar alterations were observed both in T2DM and AD within this pathway. Such molecular alterations drive the impairment of glucose uptake and metabolism as well as an increase of inflammatory processes within the brain and likely suggest that PI3K/Akt axis impairment could be a common denominator in those diseases.

Interestingly, the alteration of the PI3K/Akt axis in $\mathrm{AD}$ may result from accumulation of cholesterol oxidation products in the brain called oxysterols. Some oxysterols (e.g., 27-OHC, 7 $\beta$ $\mathrm{OHC}$, and 7-KC) deriving from cholesterol enzymatic oxidation or auto-oxidation further exacerbate cell-damage by sustaining free-radical chain reactions (Palozza et al., 2008; Niki, 2018). Furthermore, increased oxysterols levels were detected in $\mathrm{AD}$ brains compared to controls (Hascalovici et al., 2009; Testa et al., 2016). Gamba et al. address this topic by discussing how cholesterol oxidation products, i.e., oxysterols, may favor brain insulin resistance development, thus contributing to disrupt glucose uptake resulting in increased accumulation and reduced clearance of both $\mathrm{A} \beta$ and phospho-Tau in $\mathrm{AD}$ brain.

Similarly, Chatterjee et al. provide experimental evidence that the impairment of the PI3K/Akt axis due to insulin resistance favors Tau phosphorylation through a mechanism involving GSK3 $\beta$ and reduced autophagy in Drosophila. These authors show that co-expression of Chico (homolog of the mammalian IRS) and Tau leads to GSK3 $\beta$ inactivation and reduces Tau hyperphosphorylation in Drosophila. Conversely, the co-expression of insulin-resistant Chico loss of function results in hyper-active GSK3 $\beta$ and Tau hyperphosphorylation, thus suggesting that IRS1 would play a pivotal role in controlling downstream kinases in $\mathrm{AD}$.

The detrimental impact of metabolic disorders, e.g., T2DM, obesity and metabolic syndrome, on brain structure and function has been also addressed. A research paper authored by Kavanagh et al. provides evidence that T2DM in vervet monkeys produces alterations in brain metabolism that foster the amyloidogenic pathway similar to what is observed in pre-symptomatic AD. This study shows that during the progression from healthy to pre-diabetes to T2DM, the brain moves into a state of altered metabolism that is characterized by higher glucose and lower amino acids and acylcarnitines levels. Then, increased cerebral metabolism seems to drive $A \beta$ production and accelerates $A \beta$ aggregation, in T2D similar to AD. These results shed light on the mechanisms through which T2DM development could lead to $\mathrm{AD}$-related pathology and cognitive decline.

Movassat et al. briefly examined the main mechanisms linking $\mathrm{T} 2 \mathrm{DM}$ to $\mathrm{AD}$ and provide the first evidence that certain circulating AD biomarkers can be found in goto-kakizaki (GK) rats, a model of non-obesity induced diabetes, suggesting that GK rats may be a model to investigate common molecular 
mechanisms of both disorders. Furthermore, Duarte et al. tested the hypothesis that caffeine exposure ameliorates T2DMinduced hippocampal alterations in GK rat brain. Caffeine is a non-selective antagonist of adenosine receptors (both $A_{1} R$ and $A_{2 A} R$ ), whose activity impact on the molecular processes regulating cognitive and learning functions. $A_{1} R$ and $A_{2 A} R$ were found to be altered in the brain of T2D animal models. Caffeine-mediated neuroprotective effects were likely promoted through the reduction of $\mathrm{A}_{2 \mathrm{~A}}$ Rs activity at the synaptic level as well as in glial cells. Indeed, caffeine long-term intake was associated with improved memory functions, reduced astrogliosis, and reduced hippocampal synaptic degeneration in GK rats. Although long-term intake of caffeine did not prevent T2D-induced metabolic alterations in the hippocampus, its neuroprotective effects may be of help to delay the progression of T2D-related neurodegeneration.

With regard to obesity, Lloret et al. discuss the increased risk to develop $\mathrm{AD}$ for overweight and obese individuals, describing the role of obesity-associated hyper-leptinemia in promoting brain insulin resistance and glutamate-induced excitotoxicity. Under physiological conditions, insulin secretion stimulates leptin synthesis and release by adipocytes thus favoring satiety, whereby, as in a vicious cycle, leptin reduces insulin secretion and enhances insulin sensitivity to promote glucose uptake and metabolism. Moreover, leptin was shown to have neuroprotective functions by favoring long-term potentiation (LTP) and boosting the activity of $\mathrm{N}$-methyl-D-aspartate (NMDA) receptors at synaptic levels. Conversely, increased circulating leptin levels would lead to leptin resistance and consequently to insulin resistance found to be associated with LTP dysfunction, and NMDA excitotoxicity in obese individuals and AD subjects. For that reasons, obesity in middle-age could be considered as a risk factor to develop AD in the elderly.

Among the intracellular processes dependent on the rate of glucose uptake, that are altered under insulin resistance conditions, O-GlcNAcylation post-translational modifications emerged as a key process regulating protein functions (Moll et al., 2020; Zuliani et al., 2021). Ansari and Emerald discuss the reciprocal interaction between insulin signaling and $\mathrm{O}$ GlcNAcylation process in the brain, highlighting that increased O-GlcNAcylation of active sites of proteins of insulin signaling may promote the development of brain insulin resistance. In turn, brain insulin resistance, by reducing glucose uptake, likely prevent the O-GlcNAcylation process, thus impairing the activity of many proteins. In particular, reduced $\mathrm{O}$ GlcNAcylation of amyloid precursor protein (APP) and Tau may be responsible for increased $A \beta$ production as well as Tau phosphorylation, both processes associated with the development of AD.

Another key aspect that links metabolic disorders and development of neurodegenerative diseases is the lipid dysmetabolism (Trostchansky, 2019; Falabella et al., 2021). Indeed, insulin signaling, beyond glucose metabolism, also regulates lipid metabolism while insulin resistance leads to dyslipidemia (Arnold et al., 2018). By discussing current evidence, Le Stunff et al. propose that an excess of toxic lipids generated in the liver can be a cause of neurodegeneration. In particular, dyslipidemia may lead to increased ceramide levels that, due to their hydrophobic nature, can cross the BBB, thus promoting an exaggerate production of pro-inflammatory cytokines within the brain fostering development of brain insulin resistance and cognitive decline.

The relevance for brain insulin resistance in development of Parkinson disease (PD) was also described. Fiory et al. highlight how peripheral alterations, such those occurring in T2DM, have detrimental effects on PD, by negatively affecting PD phenotype, accelerating its progression and worsening cognitive impairment. These authors provide an extensive analysis of the recent lines of evidence supporting this idea, by showing how insulin resistance both in peripheral tissues and in the brain worsens functions of dopaminergic neurons, favors alphasynuclein aggregation, impairs mitochondrial functions and promotes a pro-inflammatory state, that are all features of PD. Moreover, they provide an update about the neuroprotective effects of antidiabetic drugs on PD onset and progression collected both in humans and animal models.

Among the strategies to overcome T2DM-associated metabolic dysfunctions and cognitive decline the role of glucagon-like peptide-1 (GLP1) is gaining much attention. Indeed, GLP1 and insulin pathway share several targets downstream from IRS1, including Akt and MAPK, thus meaning that activation of GLP1 cascade may be useful to overcome IRS1 inhibition and thus insulin resistance (Tramutola et al., 2017; Holscher, 2019). Grieco et al. address this aspect by emphasizing how the increased activation of GLP1 signaling pathway obtained through the administration of GLP1 receptor (GLP1R) agonists in experimental models of $\mathrm{AD}, \mathrm{PD}$ and T2DM promotes neuroprotective effects. These latter include reduced $A \beta$ and hyperphosphorylated Tau, reduced oxidative stress and inflammatory processes and improved functions of dopaminergic neurons. Overall, by reducing neurotoxic events, GLP1R agonists ameliorate synaptic plasticity thus exerting beneficial effects on cognitive functions.

Finally, Dierssen et al. provide a comprehensive analysis of the literature addressing the role of insulin signaling in DS. DS is the most frequent chromosomal abnormality responsible for intellectual disability, due to the presence of an extra complete or segment of chromosome 21 (Hsa21). Furthermore, $\mathrm{DS}$ individuals are at high risk to develop $\mathrm{AD}$ after the age of 40. Multiple genes and factors are responsible for the major DS phenotypes and as explained in the review, insulin signaling in the brain is thought to mediate brain dysfunction associated with intellectual disability and the development of AD in DS.

\section{AUTHOR CONTRIBUTIONS}

All authors listed have made a substantial, direct and intellectual contribution to the work, and approved it for publication. 


\section{REFERENCES}

Arnold, S. E., Arvanitakis, Z., Macauley-Rambach, S. L., Koenig, A. M., Wang, H. Y., Ahima, R. S., et al. (2018). Brain insulin resistance in type 2 diabetes and Alzheimer disease: concepts and conundrums. Nat. Rev. Neurol. 14, 168-181. doi: 10.1038/nrneurol.2017.185

Barone, E., Di Domenico, F., Cassano, T., Arena, A., Tramutola, A., Lavecchia, M. A., et al. (2016). Impairment of biliverdin reductase-A promotes brain insulin resistance in Alzheimer disease: a new paradigm. Free Radic. Biol. Med. 91, 127-142. doi: 10.1016/j.freeradbiomed.2015.12.012

Barone, E., Mosser, S., and Fraering, P. C. (2014). Inactivation of brain Cofilin1 by age, Alzheimer's disease and gamma-secretase. Biochim. Biophys. Acta 1842(12 Pt A), 2500-2509. doi: 10.1016/j.bbadis.2014.10.004

Barone, E., Tramutola, A., Triani, F., Calcagnini, S., Di Domenico, F., Ripoli, C., et al. (2019). Biliverdin reductase-a mediates the beneficial effects of intranasal insulin in Alzheimer disease. Mol. Neurobiol. 56, 2922-2943. doi: 10.1007/s12035-018-1231-5

Bomfim, T. R., Forny-Germano, L., Sathler, L. B., Brito-Moreira, J., Houzel, J. C., Decker, H., et al. (2012). An anti-diabetes agent protects the mouse brain from defective insulin signaling caused by Alzheimer's disease- associated Abeta oligomers. J Clin Invest. 122, 1339-1353. doi: 10.1172/JCI57256

Brás, I. C., König, A., and Outeiro, T. F. (2019). Glycation in Huntington's disease: a possible modifier and target for intervention. J. Huntingtons Dis. 8, 245-256. doi: $10.3233 / J H D-190366$

Butterfield, D. A., Di Domenico, F., and Barone, E. (2014). Elevated risk of type 2 diabetes for development of Alzheimer disease: a key role for oxidative stress in brain. Biochim. Biophys. Acta 1842, 1693-1706. doi: 10.1016/j.bbadis.2014.06.010

de la Monte, S. M. (2019). The full spectrum of Alzheimer's disease is rooted in metabolic derangements that drive type 3 diabetes. Adv. Exp. Med. Biol. 1128, 45-83. doi: 10.1007/978-981-13-3540-2_4

Dearden, L., Buller, S., Furigo, I. C., Fernandez-Twinn, D. S., and Ozanne, S. E. (2020). Maternal obesity causes fetal hypothalamic insulin resistance and disrupts development of hypothalamic feeding pathways. Mol. Metab. 42:101079. doi: 10.1016/j.molmet.2020.101079

Falabella, M., Vernon, H. J., Hanna, M. G., Claypool, S. M., and Pitceathly, R. D. S. (2021). Cardiolipin, mitochondria, and neurological disease. Trends Endocrinol. Metab. 32, 224-237. doi: 10.1016/j.tem.2021. 01.006

Franklin, W., Krishnan, B., and Taglialatela, G. (2019). Chronic synaptic insulin resistance after traumatic brain injury abolishes insulin protection from amyloid beta and tau oligomer-induced synaptic dysfunction. Sci. Rep. 9:8228. doi: 10.1038/s41598-019-44635-Z

Fusco, S., Spinelli, M., Cocco, S., Ripoli, C., Mastrodonato, A., Natale, F., et al. (2019). Maternal insulin resistance multigenerationally impairs synaptic plasticity and memory via gametic mechanisms. Nat. Commun. 10:4799. doi: 10.1038/s41467-019-12793-3

Hascalovici, J. R., Vaya, J., Khatib, S., Holcroft, C. A., Zukor, H., Song, W., et al. (2009). Brain sterol dysregulation in sporadic AD and MCI: relationship to heme oxygenase-1. J. Neurochem. 110, 1241-1253. doi: 10.1111/j.1471-4159.2009.06213.x

Holscher, C. (2019). Insulin signaling impairment in the brain as a risk factor in Alzheimer's disease. Front. Aging Neurosci. 11:88. doi: $10.3389 /$ fnagi.2019.00088

Hong, C. T., Chen, K. Y., Wang, W., Chiu, J. Y., Wu, D., Chao, T. Y., et al. (2020). Insulin resistance promotes parkinson's disease through aberrant expression of alpha-synuclein, mitochondrial dysfunction, and deregulation of the polo-like kinase 2 signaling. Cells 9:740. doi: 10.3390/cells9030740

Kellar, D., and Craft, S. (2020). Brain insulin resistance in Alzheimer's disease and related disorders: mechanisms and therapeutic approaches. Lancet Neurol. 19, 758-766. doi: 10.1016/S1474-4422(20)30231-3

Kleinridders, A., Cai, W., Cappellucci, L., Ghazarian, A., Collins, W. R., Vienberg, S. G., et al. (2015). Insulin resistance in brain alters dopamine turnover and causes behavioral disorders. Proc. Natl. Acad. Sci. U.S.A. 112, 3463-3468. doi: 10.1073/pnas.1500877112

Lanzillotta, C., Tramutola, A., Di Giacomo, G., Marini, F., Butterfield, D. A., Di Domenico, F., et al. (2021). Insulin resistance, oxidative stress and mitochondrial defects in Ts65dn mice brain: a harmful synergistic path in down syndrome. Free Radic. Biol. Med. 165, 152-170. doi: 10.1016/j.freeradbiomed.2021.01.042

Lourenco, M. V., Clarke, J. R., Frozza, R. L., Bomfim, T. R., Forny-Germano, L., Batista, A. F., et al. (2013). TNF-alpha mediates PKR-dependent memory impairment and brain IRS-1 inhibition induced by Alzheimer's beta-amyloid oligomers in mice and monkeys. Cell Metab. 18, 831-843. doi: 10.1016/j.cmet.2013.11.002

Manco, M., Guerrera, S., Ravà, L., Ciofi Degli Atti, M., Di Vara, S., Valeri, G., et al. (2021). Cross-sectional investigation of insulin resistance in youths with autism spectrum disorder. Any role for reduced brain glucose metabolism? Transl Psychiatry. 11:229. doi: 10.1038/s41398-021-01345-3

Melo, H. M., Seixas da Silva, G. D. S., Sant'Ana, M. R., Teixeira, C. V. L., Clarke, J. R., et al. (2020). Palmitate is increased in the cerebrospinal fluid of humans with obesity and induces memory impairment in mice via pro-inflammatory TNF-alpha. Cell Rep. 30, 2180.e8-2194.e8. doi: 10.1016/j.celrep.2020.01.072

Moll, T., Shaw, P. J., and Cooper-Knock, J. (2020). Disrupted glycosylation of lipids and proteins is a cause of neurodegeneration. Brain 143, 1332-1340. doi: 10.1093/brain/awz358

Nakabeppu, Y. (2019). Molecular pathophysiology of insulin depletion, mitochondrial dysfunction, and oxidative stress in Alzheimer's disease brain. Adv. Exp. Med. Biol. 1128, 27-44. doi: 10.1007/978-981-13-3540-2_3

Niki, E. (2018). Oxidant-specific biomarkers of oxidative stress. Association with atherosclerosis and implication for antioxidant effects. Free Radic. Biol. Med. 120, 425-440. doi: 10.1016/j.freeradbiomed.2018.04.001

Palozza, P., Barone, E., Mancuso, C., and Picci, N. (2008). The protective role of carotenoids against 7-keto-cholesterol formation in solution. Mol. Cell Biochem. 309, 61-68. doi: 10.1007/s11010-007-9643-y

Rhea, E. M., Raber, J., and Banks, W. A. (2020). ApoE and cerebral insulin: trafficking, receptors, and resistance. Neurobiol. Dis. 137:104755. doi: 10.1016/j.nbd.2020.104755

Sharma, N., Tramutola, A., Lanzillotta, C., Arena, A., Blarzino, C., Cassano, T., et al. (2019). Loss of biliverdin reductase-A favors Tau hyperphosphorylation in Alzheimer's disease. Neurobiol. Dis. 125, 176-189. doi: $10.1016 /$ j.nbd.2019.02.003

Spinelli, M., Fusco, S., Mainardi, M., Scala, F., Natale, F., Lapenta, R., et al. (2017). Brain insulin resistance impairs hippocampal synaptic plasticity and memory by increasing GluA1 palmitoylation through FoxO3a. Nat. Commun. 8:2009. doi: 10.1038/s41467-017-02221-9

Talbot, K., Wang, H. Y., Kazi, H., Han, L. Y., Bakshi, K. P., Stucky, A., et al. (2012). Demonstrated brain insulin resistance in Alzheimer's disease patients is associated with IGF-1 resistance, IRS-1 dysregulation, and cognitive decline. J. Clin. Invest. 122, 1316-1338. doi: 10.1172/JCI59903

Testa, G., Staurenghi, E., Zerbinati, C., Gargiulo, S., Iuliano, L., Giaccone, G., et al. (2016). Changes in brain oxysterols at different stages of Alzheimer's disease: their involvement in neuroinflammation. Redox. Biol. 10, 24-33. doi: 10.1016/j.redox.2016.09.001

Tramutola, A., Arena, A., Cini, C., Butterfield, D. A., and Barone, E. (2017). Modulation of GLP-1 signaling as a novel therapeutic approach in the treatment of Alzheimer's disease pathology. Expert Rev. Neurother. 17, 59-75. doi: 10.1080/14737175.2017.1246183

Tramutola, A., Lanzillotta, C., Arena, A., Barone, E., Perluigi, M., and Di Domenico, F. (2016). Increased mammalian target of rapamycin signaling contributes to the accumulation of protein oxidative damage in a mouse model of Down's syndrome. Neurodegener. Dis. 16, 62-68. doi: 10.1159/000441419

Tramutola, A., Lanzillotta, C., Di Domenico, F., Head, E., Butterfield, D. A., Perluigi, M., et al. (2020). Brain insulin resistance triggers early onset Alzheimer disease in Down syndrome. Neurobiol. Dis. 137:104772. doi: 10.1016/j.nbd.2020.104772

Tramutola, A., Triplett, J. C., Di Domenico, F., Niedowicz, D. M., Murphy, M. P., Coccia, R., et al. (2015). Alteration of mTOR signaling occurs early in the progression of Alzheimer disease (AD): analysis of brain from subjects with pre-clinical $\mathrm{AD}$, amnestic mild cognitive impairment and late-stage $\mathrm{AD} . J$. Neurochem. 133, 739-749. doi: 10.1111/jnc.13037

Triani, F., Tramutola, A., Di Domenico, F., Sharma, N., Butterfield, D. A., Head, E., et al. (2018). Biliverdin reductase-A impairment links brain insulin resistance with increased Abeta production in an animal model of aging: Implications for Alzheimer disease. Biochim. Biophys. Acta Mol. Basis Dis. 1864, 3181-3194. doi: 10.1016/j.bbadis.2018.07.005 
Trostchansky, A. (2019). Overview of lipid biomarkers in amyotrophic lateral sclerosis (ALS). Adv. Exp. Med. Biol. 1161, 233-241. doi: 10.1007/978-3-030-21735-8_18

Zuliani, I., Lanzillotta, C., Tramutola, A., Barone, E., Perluigi, M., Rinaldo, S., et al. (2021). High-fat diet leads to reduced protein O-GlcNAcylation and mitochondrial defects promoting the development of Alzheimer's disease signatures. Int. J. Mol. Sci. 22:3746. doi: 10.3390/ijms220 73746

Conflict of Interest: The authors declare that the research was conducted in the absence of any commercial or financial relationships that could be construed as a potential conflict of interest.
Publisher's Note: All claims expressed in this article are solely those of the authors and do not necessarily represent those of their affiliated organizations, or those of the publisher, the editors and the reviewers. Any product that may be evaluated in this article, or claim that may be made by its manufacturer, is not guaranteed or endorsed by the publisher.

Copyright (c) 2021 Dierssen and Barone. This is an open-access article distributed under the terms of the Creative Commons Attribution License (CC BY). The use, distribution or reproduction in other forums is permitted, provided the original author(s) and the copyright owner(s) are credited and that the original publication in this journal is cited, in accordance with accepted academic practice. No use, distribution or reproduction is permitted which does not comply with these terms. 